\title{
DESTRUCCIÓN DEL PATRIMONIO ARQUITECTÓNICO Y MODIFICACIONES NORMATIVAS EN CONTEXTO SÍSMICO. El caso del terremoto-tsunami de Cobquecura, Chile $2010^{1}$.
}

\author{
Antonio Sahady Villanueva \\ Facultad de Arquitectura y Urbanismo \\ Universidad de Chile \\ asahady@uchilefau.cl \\ Mario Ferrada Aguilar \\ Facultad de Arquitectura y Urbanismo \\ Universidad de Chile \\ mferrada@uchilefau.cl
}

\section{RESUMEN}

En el territorio americano, Chile es uno de los países más expuestos a los desastres naturales. Particularmente, a los movimientos telúricos. $Y$ aun cuando esta realidad es insoslayable, no se advierten cambios manifiestos en el ámbito de políticas públicas, que consideren los riesgos de desastre sísmico en el campo del patrimonio cultural y arquitectónico. Esta ausencia impide abordar integralmente las tareas de prevención y reconstrucción de este patrimonio, imposibilitando actuar en la disminución de los factores del riesgo y en el control de la vulnerabilidad de la arquitectura y los lugares de valor. Sobre esta base se revisa la respuesta del Estado ante los efectos causados por los grandes sismos del último medio siglo. Especial atención tiene el examen de los cambios legales y normativos, en materia de patrimonio arquitectónico y urbano, a raíz del terremoto y tsunami del año 2010, ocurrido al sur de Chile.

Palabras clave: Patrimonio arquitectónico, Riesgos sísmicos, Normativa.

Bloque temático: Teoría e historia de la ciudad.

\begin{abstract}
In the American territory, Chile is one of the countries most exposed to natural disasters. Particularly, to seismic movements. And even when this reality is unavoidable, there are no obvious changes in the field of public policies, which consider the risks of seismic disaster in the field of cultural and architectural heritage. This absence makes it impossible to comprehensively address the tasks of prevention and reconstruction of this heritage, making it impossible to act in the reduction of risk factors and in the control of the vulnerability of architecture and places of value. On this basis, the State's response to the effects caused by the great earthquakes of the last half century is reviewed. Special attention is paid to the legal and regulatory changes in terms of architectural and urban heritage, following the earthquake and tsunami of 2010, which occurred in southern Chile.
\end{abstract}

Keywords: Architectural heritage, seismic risks, regulations.

Topic: Theory and history of the city.

\footnotetext{
${ }^{1}$ Artículo derivado del Proyecto de Investigación FAU 2.0/2018-2019 "Relato audiovisual de los terremotos y las políticas públicas del patrimonio arquitectónico y urbano de Chile. 1971-2017". Investigador responsable Mario Ferrada Aguilar. Coinvestigadores Antonio Sahady Villanueva y Max Aguirre González. Financiamiento Dirección de Investigación y Desarrollo, Facultad de Arquitectura y Urbanismo, Universidad de Chile.
} 


\section{Introducción}

Durante los siglos $X X$ y $X X I$, en Chile se han sucedido 22 grandes terremotos por sobre los 7 grados de magnitud Richter. Gran parte de ellos han venido acompañados de tsunamis. En el siglo XX se registraron 16 de ellos, y hasta la fecha, en el siglo XXI se contabilizan 6 megasismos que superaron los 7,6 grados de magnitud Richter, con epicentros en los territorios del extremo norte y centro-norte (regiones de Arica y Parinacota, Tarapacá, Antofagasta y Coquimbo), zona central (regiones del Ñuble y del Biobío) y sur del país (región de Los Lagos).

El terremoto y tsunami de 2010, con epicentro en Cobquecura, actual región del Ñuble al sur del país, tuvo una magnitud de 8,8 grados Richter. El evento tuvo una extensión de 700 kilómetros, e involucró a 6 regiones de Chile, afectó al $75 \%$ de la población total del país, y su destrucción fue observada en áreas urbanas centrales y localidades rurales. Por su extensión y destrucción, es el evento de mayor impacto registrado hasta la fecha.

Fue, además el momento que evidenció la insuficiencia de instrumentos legales y normativos que atendieran al patrimonio como parte de un sistema integral del riesgo sísmico. A las pérdidas en vidas humanas y damnificados del 2010, debemos sumar la intensa destrucción de las infraestructuras viales y sanitarias, servicios públicos, la pérdida de la capacidad habitacional y la desaparición o grave daño en valiosas expresiones de la identidad arquitectónica, tanto erudita como vernácula.

A partir del sismo y tsunami de 2010, se aborda el problema de cómo las Políticas Públicas de Chile establecen mecanismos legales y normativos para la preservación del patrimonio construido, bajo el contexto del riesgo sísmico ante desastres. Las leyes, normativas y planes emanadas de esta experiencia, no han logrado hacerse cargo de los factores de riesgo y del control de los niveles de vulnerabilidad del patrimonio, el que involucra no solo la dimensión construida y física, sino también la social.

El objetivo del trabajo consiste en develar el efecto que tuvo el terremoto y tsunami de 2010 en las políticas públicas de conservación y gestión del patrimonio arquitectónico chileno, particularmente sobre los tipos de instrumentos legales y normativos. Esta indagación produce el ambiente propicio para comprender, caracterizar y reformular las nuevas políticas públicas en el ámbito patrimonial, incorporando iniciativas que propendan a la recuperación de aquellos inmuebles y conjuntos de valor que han sido castigados por el sismo, así como disminuir a niveles posibles sus condiciones de vulnerabilidad.

Para alcanzar una respuesta clara, hace falta revisar los documentos normativos que se han elaborado en las últimas décadas al respecto de las solicitaciones dinámicas que afectan a los inmuebles en las zonas expuestas. Para precisar el marco de operaciones, se ha seleccionado el terremoto de 2010, con epicentro en la localidad de Cobquecura en la actual región de Ñuble.

Se ha aplicado una metodología de tipo exploratoria, con acento cuantitativo, sin desconocer la presencia del factor cualitativo. Los instrumentos de análisis utilizados han consistido en bases de datos y documentación técnica proporcionadas por organismos estatales: Ministerio de Vivienda y Urbanismo, Ministerio de Obras Públicas, Consejo de Monumentos Nacionales, Instituto Nacional de Estadísticas, municipalidades, además de los entes particulares involucrados. Complementariamente se han considerado reportes de prensa y registros fotográficos en torno al sismo de 2010. El objeto de estudio lo constituyen las políticas públicas, acciones y medidas implementadas por el Estado sobre el patrimonio arquitectónico afectado con dicho desastre. 


\section{Riesgo sísmico y respuesta estatal frente al patrimonio.}

Durante el siglo $\mathrm{XX}$, el mayor desarrollo de la arquitectura y la densidad construida significó también una concentración demográfica que se tradujo en aumento de los índices de muertes, hacinamiento y ostensible pérdida de edificaciones. Casos patentes están representados por los desastres de 1906 (Valparaíso), 1928 (Talca), 1939 (Chillán), 1960 (Valdivia), 1971 (Illapel) y 1985 (San Antonio). En general, los sismos de esta fase suscitaron la preocupación por las normativas de construcción, los planes de reconstrucción, el diseño de la vivienda popular y masiva, y la planificación racional del crecimiento urbano. Los terremotos de este periodo contribuyeron a elevar el desarrollo urbano de las zonas afectadas (Ferrada, 2017).

Un ejemplo de ese avance: en 1929, un año después del terremoto de Talca, se promulgó la Ley $\mathrm{N}^{\circ} 4.563$ sobre "Construcciones antisísmicas", que constituyó la base de la promulgación en 1935 de la Ley y Ordenanza General de Construcciones y Urbanización, en cuyo cuerpo se institucionalizaron las primeras normas de diseño y construcción antisísmicas de Chile (Arenas Et Al, 2010). Poco después, en 1931 se dictó el Decreto con Fuerza de Ley $N^{\circ} 345$, en el cual se dictó la Ley General y se incluyó la Ordenanza General de Urbanismo y Construcción. Ambas normas empezaron a regir a contar de 1936, año en el cual fueron publicadas en el Diario Oficial (Fernández; Holmes 2009).

Entre fines del siglo XX e inicios del XXI, los eventos sísmicos observados muestran cambios cualitativos en la respuesta del Estado, respecto de lo sucedido hasta 1985. En este periodo los terremotos se concentraron en la zona norte de Chile: Punitaqui (1997), Tarapacá (2005), Tocopilla (2007), Cobquecura (2010), Iquique (2014), Coquimbo (2015). En el sur del país: Chiloé (2016). En esta etapa surge incipientemente un nuevo enfoque, basado en la gestión integral de los desastres, principalmente de origen sísmico, visibilizando los riesgos en el territorio, las estructuras construidas y la población.

Los desastres se presentan cuando se desencadena "una fuerza con potencial destructivo (amenaza), $y$ encuentra condiciones de debilidad ante esa fuerza, o incapacidad para oponerse a sus efectos (vulnerabilidad)" (Vargas, 2002). Los desastres son fenómenos sociales, ya que los sucesos naturales devienen en desastres solamente cuando afectan a las personas; comportan, por tanto, destrucción de vidas humanas, del medio y de las condiciones de subsistencia.

En la idea de confeccionar políticas públicas acertadas, en materia de desastres deben enfocarse tanto en las amenazas como en la vulnerabilidad de las comunidades expuestas, abordando las acciones de la prevención, la mitigación y la reconstrucción. Significa esto, orientarse hacia una concepción holística del riesgo, que considere no sólo las variables geológicas y estructurales. También son fundamentales las económicas, sociales, políticas y culturales (Cardona, 2001)

En esta medida, el desastre sísmico menoscaba el sistema de valores que entrañan los bienes del patrimonio chileno, sea este local, nacional y/o mundial. Como se sabe, el riesgo de desastre es producto de la amenaza y la vulnerabilidad. Una amenaza es un fenómeno (como un terremoto) que encierra el potencial de causar trastornos a los bienes culturales. La vulnerabilidad, a su vez, es la exposición de un bien cultural a la amenaza. Mientras que la amenaza es la fuente externa de un desastre, la vulnerabilidad es la debilidad intrínseca del bien (puede deberse a su ubicación o a sus características específicas). Por consiguiente, es posible prevenir o, como mínimo, reducir considerablemente sus efectos, reforzando la resiliencia de los bienes del patrimonio que deben preservarse, incorporándolos en las políticas públicas, a través de planes de manejo de riesgos.

Aunque el patrimonio no suele incluirse en las estadísticas mundiales sobre el riesgo de desastres, los bienes culturales y naturales se ven crecientemente afectados por fenómenos que son cada vez menos "naturales" en 
sus dinámicas, y también en su causa. La pérdida progresiva de esos bienes por terremotos, inundaciones, aludes de lodo, incendios, disturbios civiles y otros peligros, se ha convertido en un gran motivo de preocupación: el patrimonio es un poderoso factor en la cohesión social y el desarrollo sostenible, sobre todo en momentos de tensión (UNISDR, 2002).

Los efectos de un desastre en los bienes culturales y naturales suelen superar con creces el deterioro causado por el desgaste paulatino a largo plazo y, en algunas ocasiones, puede llevar a su completa desaparición. Se ha demostrado que si el patrimonio se mantiene adecuadamente, puede contribuir positivamente a la reducción del riesgo de desastres.

\begin{tabular}{|c|c|c|c|c|c|}
\hline \multicolumn{6}{|c|}{ HITOS DE DESASTRES SÍSMICOS EN CHILE } \\
\hline \multicolumn{6}{|c|}{ SIGLO XX } \\
\hline & Región & Epicentro del sismo & \multicolumn{2}{|l|}{ Fecha } & $\begin{array}{l}\text { Magnitud } \\
\text { Richter }\end{array}$ \\
\hline 1 & Región de Valparaíso & Valparaíso & 16 de agosto & 1906 & $8.2(*)$ \\
\hline 2 & Región de Atacama & Copiapó & 4 de diciembre & 1918 & 8.2 \\
\hline 3 & Región de Atacama & Huasco & 10 de noviembre & 1922 & $8.5(*)$ \\
\hline 4 & Región del Maule & Talca & 1 de diciembre & 1928 & 8.3 \\
\hline 5 & Región del Bío Bío & Chillán & 24 de enero & 1939 & 8.3 \\
\hline 6 & Región de Coquimbo & Illapel & 6 de abril & 1943 & 8.2 \\
\hline 7 & Región de Magallanes y la Anta & Punta Arenas & 17 de diciembre & 1949 & 7.8 \\
\hline 8 & Región del Bío Bío & Chillán & 6 de mayo & 1953 & 7.6 \\
\hline 9 & Región del Bío Bío & Concepción & 21 de mayo & 1960 & 7.3 \\
\hline 10 & Región de Los Ríos & Valdivia & 22 de mayo & 1960 & $9.5(*)$ \\
\hline 11 & Región de Valparaíso & La Ligua & 28 de marzo & 1965 & 7.4 \\
\hline 12 & Región de Antofagasta & Tal Tal & 28 de diciembre & 1966 & 7.8 \\
\hline 13 & Región de Coquimbo & Illapel & 8 de julio & 1971 & 7.5 \\
\hline 14 & Región de Valparaíso & San Antonio & 3 de marzo & 1985 & $8.0(*)$ \\
\hline 15 & Región de Antofagasta & Antofagasta & 30 de julio & 1995 & $8.0\left(^{*}\right)$ \\
\hline 16 & Región de Coquimbo & Punitaqui & 14 de octubre & 1997 & 7.1 \\
\hline \multicolumn{6}{|c|}{ SIGLO XXI } \\
\hline 17 & Región de Tarapacá & Tarapacá & 13 de junio & 2005 & $7.8(*)$ \\
\hline 18 & Región de Antofagasta & Tocopilla & 14 de noviembre & 2007 & $7.7(*)$ \\
\hline 19 & Región del Maule & Constitución & 27 de febrero & 2010 & $8.8(*)$ \\
\hline 20 & Región de Arica y Parinacota & Arica & 1 de abril & 2014 & $8.2\left(^{*}\right)$ \\
\hline 21 & Región de Coquimbo & Canela & 16 de septiembre & 2015 & $8.4\left(^{*}\right)$ \\
\hline 22 & Región de Los Lagos & Chiloé & 25 de diciembre & 2016 & $7.6\left(^{*}\right)$ \\
\hline
\end{tabular}

Fig. 1. Cuadro sinóptico de desastres sísmicos ocurridos en Chile durante los siglos XX y XXI. El color destaca aquellos que por su magnitud e impacto, significaron cambios importantes en las respuestas del Estado e incidieron en los procesos de reconstrucción de la arquitectura y la ciudad. Fuente: Elaboración propia (2019).

\section{Sismo-tsunami de 2010: Desastre y respuesta estatal frente al patrimonio.}

\subsection{La destrucción y la respuesta estatal.}

El terremoto y tsunami del 27 de febrero de 2010 ha sido el más potente y destructor acaecido en Chile. El epicentro se localizó en Cobquecura, actual región de Ñuble, alcanzando una magnitud de 8,8 grados Richter². El destructivo evento trajo consigo tres imágenes escalofriantes: El edificio Alto Río en Concepción,

\footnotetext{
${ }^{2}$ Corresponde a uno de los desastres de mayor importancia ocurridos en la historia sísmica reciente de Chile, y uno de los cinco más fuertes registrados mundialmente, situándolo al nivel del evento de 1960 que destruyó la ciudad chilena de Valdivia, considerando el mayor sismo registrado en el planeta a la fecha.
} 
literalmente partido en dos, la desaparición completa de poblados costeros a causa del tsunami y el colapso de arquitecturas tradicionales, a base de adobe y madera en las localidades del interior. Los efectos más dañinos, se observaron críticamente en las regiones de O’higgins, del Maule y del Biobío. Sin embargo, la destrucción fue posible observarla también en las regiones de Valparaíso y Metropolitana.

Para los edificios y conjuntos patrimoniales, la desventura sísmica fue mayor, toda vez que se trataba de bienes irrecuperables una vez que se pierden, en el entendido que la reconstrucción no es una opción válida. Menos aun cuando no existe mano de obra especializada para intervenir, ya sea a nivel preventivo o restaurador edificios o conjuntos de valor histórico o arquitectónico. Este hecho no hizo otra cosa que poner en evidencia las debilidades normativas e institucionales en el ámbito de la arquitectura patrimonial.

Según el Plan de Reconstrucción elaborado por el Gobierno de Chile (2010) para afrontar este evento, los daños generados con la catástrofe se debieron a tres causas: a) La gran extensión geográfica que cubrió, b) El efecto que causó en todos los sectores de economía nacional, y c) la variedad de niveles de gravedad identificados

A diferencia de otros planes post sismo, el de 2010, señala que la reconstrucción debe referirse no sólo a materias de recuperación de los aspectos físicos dañados. Debe también orientarse "a restaurar medios de vida, hábitat, integración social, gobernabilidad y sostenibilidad" (Gobierno de Chile, 2010). Agregaba que la reconstrucción es "una oportunidad para planificar el futuro y conservar el pasado: la reconstrucción debe estar orientada a corregir factores de vulnerabilidad, mantener el patrimonio cultural y fortalecer la planificación del desarrollo considerando la variable de riesgo".

En dicho contexto, el "Plan de Reconstrucción MINVU. Chile Unido Reconstruye Mejor", explicitó tres programas de acción, conteniendo ciertas variaciones respecto de lo señalado por el Gobierno en su Plan de abril 2010, a saber: a) Programa de reconstrucción de viviendas, b) Programa de asistencia a aldeas de emergencia y condominios sociales, y c) Programa territorial, urbano y patrimonial de reconstrucción (Ministerio de Vivienda y Urbanismo, 2011)

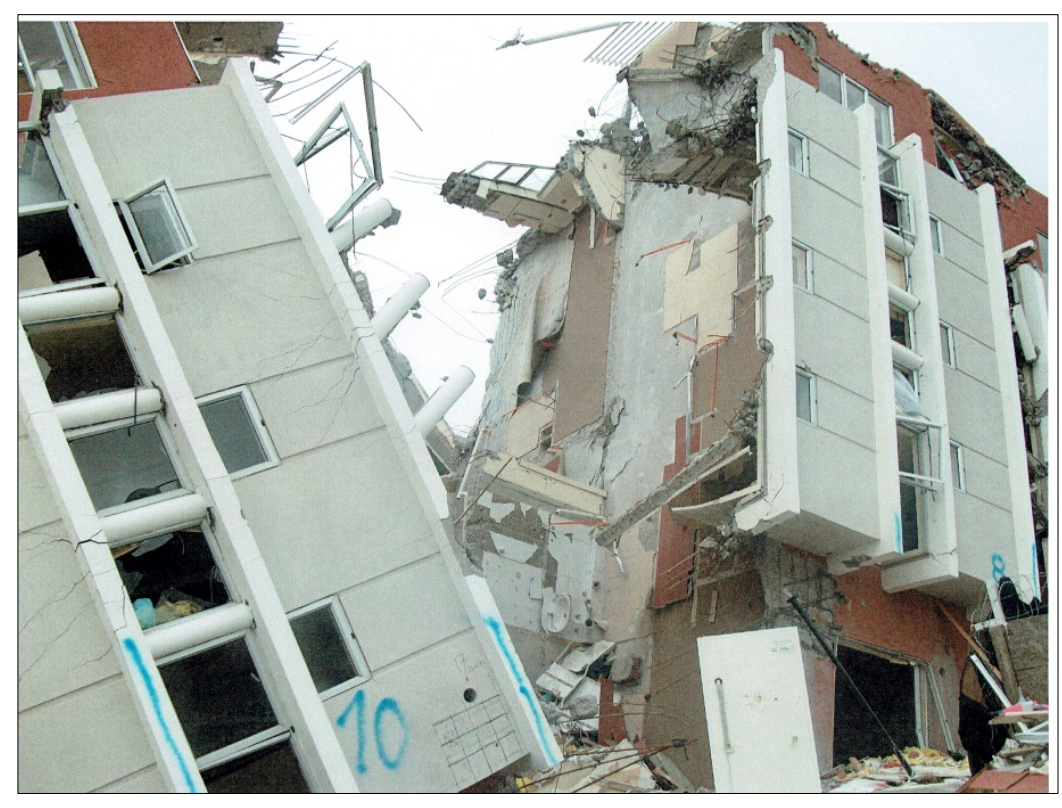

Fi. 2. Edificio Alto Río en la ciudad de Concepción. El sismo de 2010 significó el colapso completo de este edificio de viviendas de 15 pisos. Se transformó en unos de los íconos más divulgados del sismo. Fuente: Ministerio de Vivienda y Urbanismo (2010). 


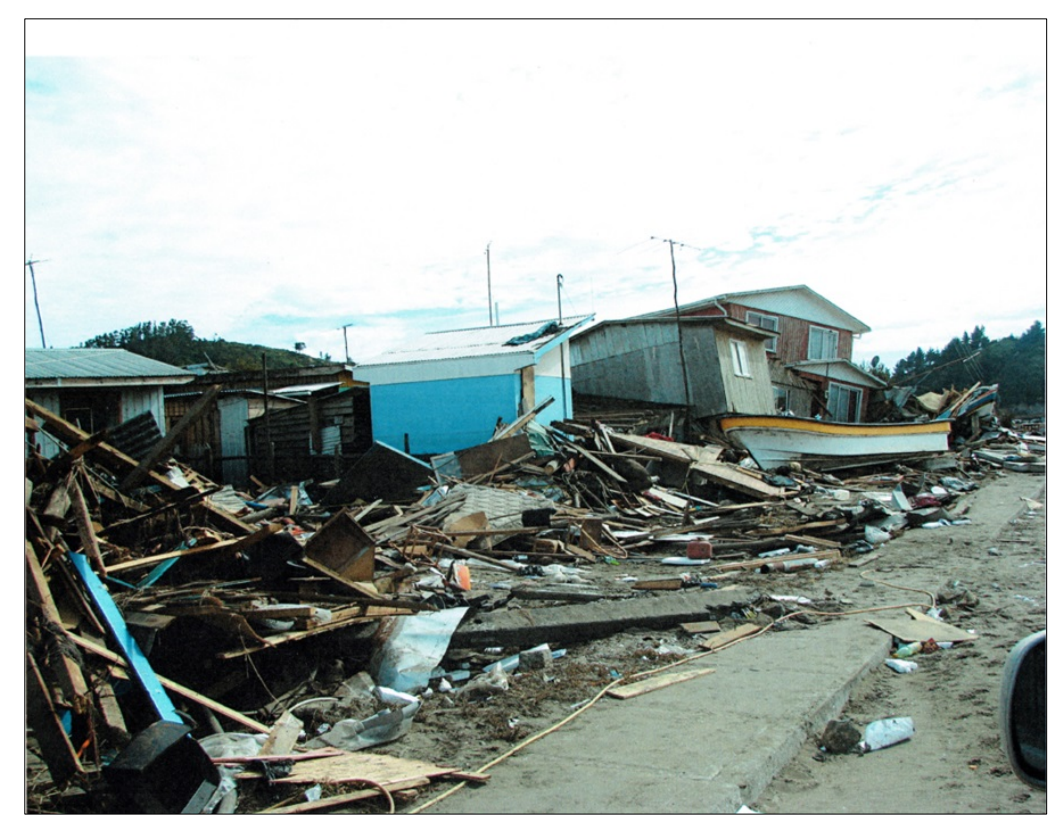

Fig. 3. Efectos del terremoto y tsunami de 2010 en el poblado y caleta de Tubul, localizado a 15 kilómetros de la ciudad de Arauco, región del Biobío. Fuente: Ministerio de Vivienda y Urbanismo (2010).

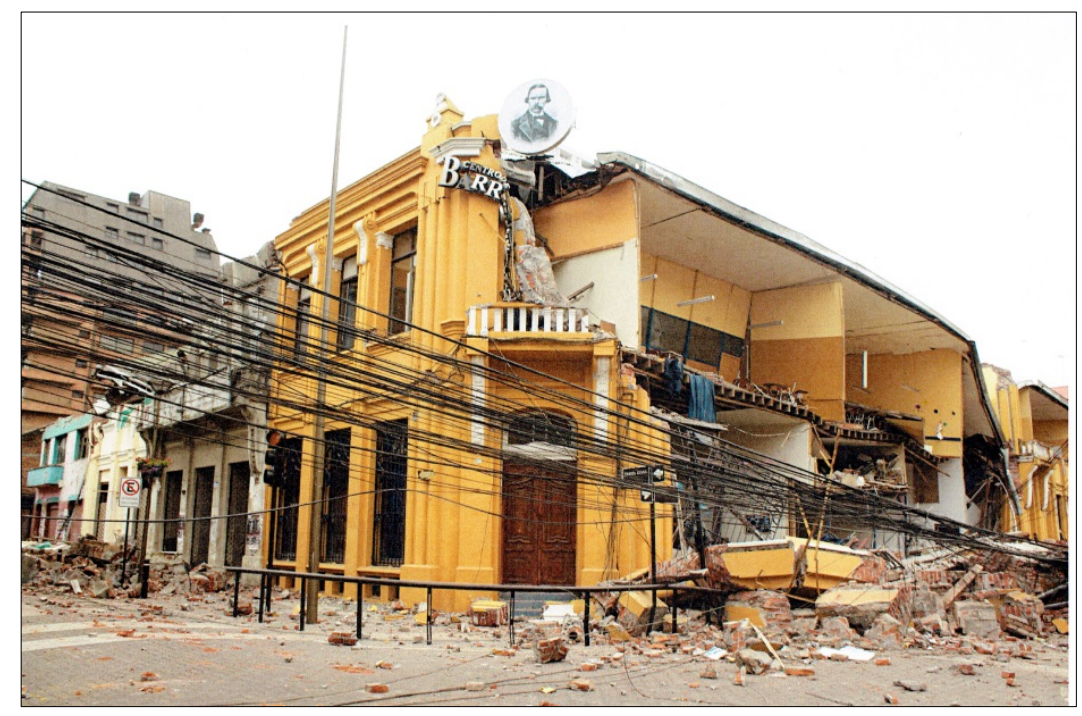

Fig. 4. Efecto del evento de 2010 en pleno centro de la ciudad de Concepción. Arquitectura de inicios del siglo XX, construida a base de entramados de madera en muros, entrepisos y techumbre. Fuente: Ministerio de Vivienda y Urbanismo (2010).

\subsection{Medidas para recuperar el patrimonio arquitectónico.}

Post desastre 2010, las medidas implementadas sobre el patrimonio arquitectónico estuvieron contextualizadas en un proceso de movimientos culturales existentes desde la década de 1990. Se había hecho explícita la necesidad de contar con una institucionalidad que diera cuenta del protagonismo de la sociedad civil y los movimientos autónomos. No es extraño entonces, que en 1991 se haya propuesto la creación de un Consejo Nacional de la Cultura y las Artes, concebido como un servicio público autónomo y descentralizado, dependiente directamente del Presidente de la República. 
En medio de este proceso, el año 2005 se creó la primera Política Cultural en Chile, en cuyos principios fundamentales se encuentra el de la "Preservación, conservación, difusión del patrimonio cultural y rescate de la memoria". Esta decisión se potenció con el terremoto y tsunami del 2010. El numeral 4 de la Política, referida a Patrimonio, Identidad y Diversidad, señala que su objetivo es "Preservar, enriquecer y difundir el patrimonio cultural del país, aumentando la inversión e implementando modernas y creativas formas de participación por parte de la comunidad".

Otra acción concurrente consistió en plantear una nueva Ley de Monumentos Nacionales, sustitutiva de la actual Ley № 17.288 de 1970, que potenciara "la conservación de los edificios con carácter histórico o artístico, apoye a los propietarios, garantice el cumplimiento de obligaciones mutuas (públicas y privadas) y promueva el reciclaje de inmuebles para evitar su obsolescencia" (CNCA, 2015). Como consecuencia del desastre de 2010, el año 2011, surgieron iniciativas para modificar esta Ley, que fue el origen del actual Ministerio de la Cultura, las Artes y el Patrimonio.

En 2013 se promulgó la modificación de la Ley N 18.985 de Donaciones Culturales, la que, en su Artículo $8^{\circ}$ incluye como nuevos beneficiarios a los dueños de inmuebles que hayan sido declarados bajo las categorías de Monumento Histórico o Zona Típica. La aprobación de esta Ley № 18.985, permitió dar un salto en materia de participación del sector privado en el financiamiento y gestión de las actividades culturales. (CNCA, 2005).

La reconstrucción post sismo-tsunami 2010 fue enfrentada por tres organismos del Estado: Ministerio del Interior con la Subsecretaría de Desarrollo Regional (SUBDERE), a través del Programa de Puesta en Valor del Patrimonio; el Consejo Nacional de la Cultura y las Artes, mediante el Programa de Apoyo a la Recuperación del Patrimonio; y, el Ministerio de Vivienda y Urbanismo, a través del Programa de Reconstrucción Patrimonial.

Particularmente, el Programa de Puesta en Valor del Patrimonio (iniciado en 2008), reorientó sus prioridades en proyectos de reconstrucción y reparaciones de inmuebles en las zonas más dañadas. La experiencia en propiedades residenciales privadas evidenció su insuficiencia, toda vez que, en general se ejecutaron trabajos de recuperación de fachadas, en situaciones donde, por los daños estructurales y deterioro espacial resultaba necesaria una restauración total e integral.

En el caso del Programa de Reconstrucción Patrimonial del Ministerio de Vivienda, buscó como objetivo recuperar la vivienda y los poblados con valor histórico, en la idea de preservar la identidad ambiental local. Mediante procesos participativos con la comunidad, se realizó el esfuerzo de reconstruir sistemas habitacionales respetando la heterogeneidad de las zonas intervenidas, incorporando diseños con patrones espaciales, morfológicos y constructivos propios del lugar.

\section{Instrumentos legales-normativos para la conservación del patrimonio arquitectónico dañado por el sismo de 2010.}

\subsection{Las normas antisísmicas en Chile.}

Es sabido que la cultura sísmica chilena ha facilitado el constante perfeccionamiento de las medidas legales y normativas sobre las estructuras sísmico-resistentes y los sistemas constructivos. Sin embargo, solamente en el último tiempo, a partir de 1990, se ha observado una incipiente tendencia en lo que respecta al patrimonio arquitectónico protegido. 
La mayoría de las construcciones nuevas salieron indemnes del desastre de 2010. No ocurrió lo mismo con aquellas más antiguas: las de adobe, escasamente mantenidas, erigidas mucho antes de que se desarrollara en Chile la legislación de construcción antisísmica, sufrieron serios colapsos. Las obras patrimoniales que sí lograron sortear con eficiencia el sismo, fueron aquellas construidas a base de entramados de madera y relleno de adobillo (fines de siglo XIX e inicios del XX), cuya resistencia y flexibilidad se han adaptado con éxito en la historia sísmica de la arquitectura chilena. Si en ellas se han visto daños, corresponden más a falta de conservación y mantenimiento preventivo adecuado, que al evento mismo.

Los edificios de valor patrimonial, lamentablemente, no pasaron la prueba con tanta eficiencia, salvo aquellas edificaciones que se han construido cumpliendo con la normativa sísmica vigente (en especial la producción arquitectónica moderna del siglo $\mathrm{XX}$ ), esto es, las que han sido intervenidas más recientemente. $\mathrm{Y}$, entre las más antiguas, aquellas que fueron concebidas con la lógica y la sabiduría de los maestros. Con todo, es justo reconocer que la norma antisísmica vigente, está diseñada para proteger la integridad de la edificación hasta donde sea posible; pero, su fin último -y el más importante- es proteger la vida humana. En cuanto a ello, las normas de diseño sísmico están encaminadas a evitar el colapso inmediato de las edificaciones ante sismos de gran magnitud, siempre pensando en el habitante.

En Chile existe normativa antisísmica. Pero no es clara la forma cómo se incorporan en ella los bienes patrimoniales. Es claro que los documentos centrales donde se concentra la normativa chilena -la Ley General de Urbanismo y Construcciones con su Ordenanza General- definen las normas técnicas NCh 433 (Norma Técnica Diseño Sísmico de Edificios) y la NCh 2369 (Norma de Diseño Sísmico de Estructuras e Instalaciones Industriales).

En 1966 se inició el estudio para crear la Norma NCh 433, la que finalmente se oficializó en 1972. El terremoto de 1985, con epicentro en San Antonio, cerca de la capital, fue la oportunidad para probar la norma. En 1996, este instrumento se actualizó a partir de la experiencia del terremoto de Antofagasta. A su vez, en 2003, se hicieron oficiales las normas técnicas NCh 2369 de Diseño Sísmico de Estructuras e Instalaciones Industriales y la NCh 2745 de Análisis y Diseño Sísmico de Edificios con Aislación Sísmica. El sismo y tsunami de 2010, ha implicado nuevas revisiones de las normas técnicas de diseño sísmico, especialmente la NCh 433.

Con todo, las normas descritas no han sido suficientes para hacer frente al flagelo sísmico nacional, porque no se encuentran vinculadas a los objetivos de preservación del patrimonio construido, a que refiere la legislación patrimonial chilena vigente (Ley $N^{\circ} 17.288$ y Art. 60 de la Ley General de Urbanismo y Construcciones). E sismo de 2010 dejó muchos problemas pendientes y lecciones por aprender. Al menos ha permitido el desarrollo de un renovado estudio de la normativa antisísmica nacional. En su afán por contribuir a la depuración de la legislación vigente, actualmente el Ministerio de Vivienda y Urbanismo elabora, a través del Instituto de la Construcción, la modificación a la norma NCh 433 y de la NCh 430 sobre Hormigón Armado. Las principales modificaciones se refieren al cálculo estructural, una vez verificados los problemas que se produjeron a raíz del evento de 2010.

Otro avance normativo de importancia, derivado del desastre de 2010 , que atañe al desarrollo urbano, lo constituye la necesidad de actualización de los planes reguladores en función de los riesgos naturales y antrópicos. Situación que "facilitara contar con normas que orientaran de forma integral la reconstrucción de los casos urbanos y localidades severamente destruidas... de manera de potenciar su desarrollo y recuperar su identidad" (Gobierno de Chile, 2010). Las áreas urbanas emplazadas en sectores costeros son las que prestan mayor atención en estos aspectos. 


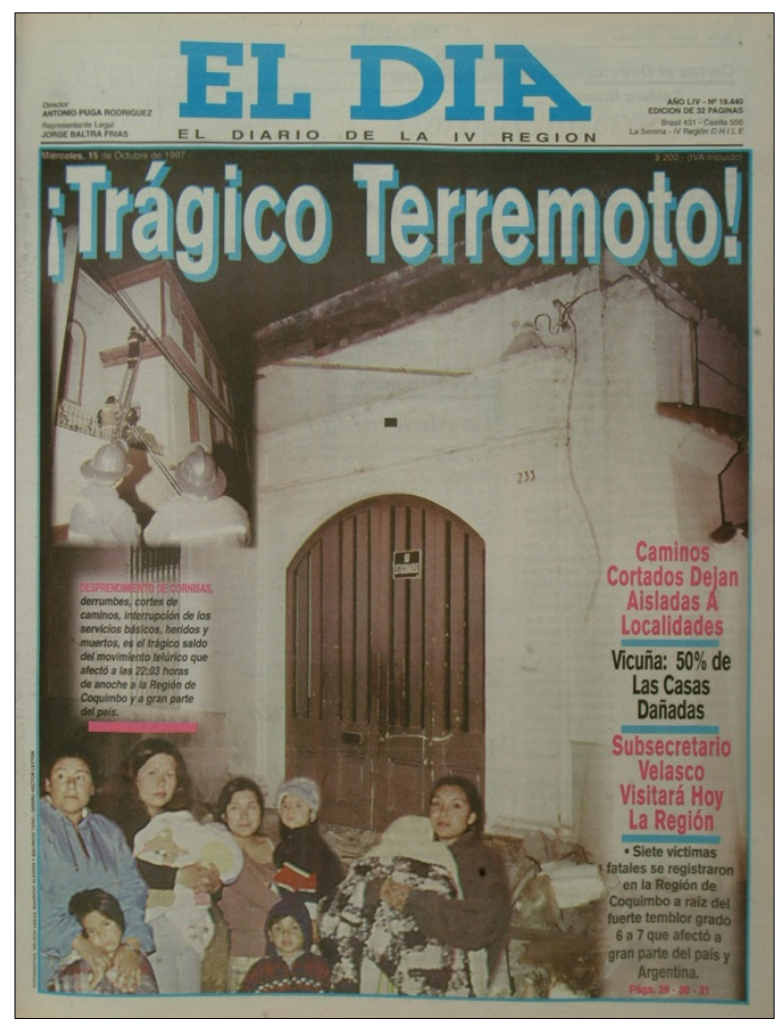

Fig. 5. La prensa informa del terremoto de Punitaqui de 1997, región de Coquimbo. En este terremoto se probaron las últimas modificaciones a la Norma NCh 433, sobre "Diseño Sísmico de Edificios". Fuente: Diario El Día de la Serena del 15 de octubre de 1997.

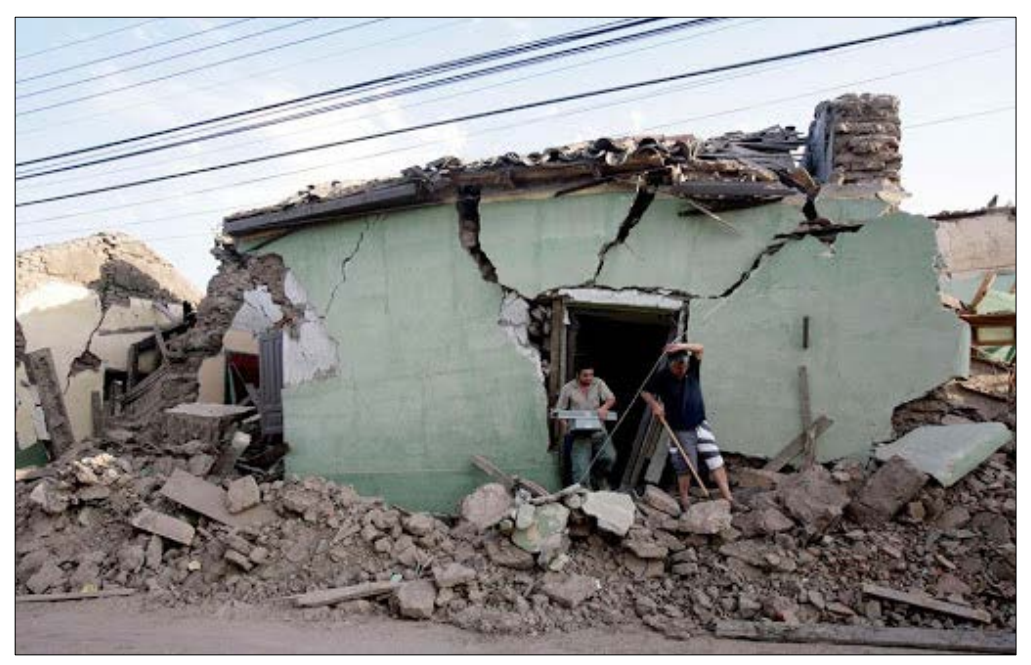

Fig. 6. Efectos en 1997 del terremoto de Punitaqui. La arquitectura popular construida en tierra y sin mayor conservación fue la más afectada. Fuente: www.guioteca.cl, (consulta 06/03/2019).

\subsection{Normas para el patrimonio vernáculo construido en tierra.}

No solo las grandes ciudades recibieron el flagelo del evento de 2010. También una multitud de pequeños poblados, mucho más indefensos y con carencias extremas vieron desaparecer o deteriorar su patrimonio, abundado la vivienda popular a base de adobe. Las opiniones respecto de cómo proceder ante las construcciones de tierra cruda en mal estado fueron variadas y a veces contradictorias. El mayor impacto sobre este tipo de patrimonio lo experimentaron las viviendas e iglesias de las zonas rurales de la región del 
O’higgins, del Maule y del Biobío, enclaves que desde la época de la Colonia han desarrollado una fuerte tradición agraria.

Hubo especialistas -arquitectos e ingenieros- que aconsejaron la demolición. Afirmaron que, por ser tan pesado y rígido, el adobe podía ser fácilmente fracturado ante cualquier acción que implicara una fuerza contra su estructura. Y de ahí a colapsar había un paso muy pequeño. Los contradictores hicieron hincapié en las cualidades del adobe como aislante térmico, lo que permite fabricar viviendas confortables y más económicas, sumado al riquísimo valor patrimonial que entrañan en nuestra cultura.

Para los poblados de interés patrimonial, dañados con el sismo-tsunami de 2010 se hizo necesario crear lo que se denominó un "polígono de interés patrimonial". Este límite referencial fue trazado por las Secretarías Regionales Ministeriales de Vivienda y Urbanismo, en acuerdo con las Direcciones de Obra de cada municipio. Se constituyó en una herramienta decisiva, que en parte vino a suplir la falta de regulación de las zonas de interés patrimonial.

Fundamentalmente estas acciones se encontraron con la dificultad de tratar la recuperación de las construcciones levantadas en tierra cruda, tema crítico en países con alta sismicidad como lo es Chile. Arquitectura caracterizada por su fragilidad, relativa baja resistencia, y expuesta a las condiciones atmosféricas, al deterioro y a la falta de mantención.

Uno de los logros más trascendentes logrados a raíz del sismo de 2010, y que han beneficiado a los poblados y la arquitectura patrimonial basada en tierra cruda, se reflejó en la aprobación en 2013 de la Norma NCh 3332, relativa a Estructuras, Intervención de construcciones patrimoniales de tierra cruda y requisitos del proyecto estructural. El objetivo de esta norma, que se comenzó a elaborar en 2009, fue el establecimiento de las bases fundamentales para ejecutar el diagnóstico correcto en la arquitectura construida en tierra cruda, identificar su condición actual y evaluar qué tipo de reparaciones y refuerzos son compatibles con sus valores históricos y su comportamiento estructural (Instituto de la Construcción, 2013)

Las edificaciones que caben en la regulación de la Norma NCh 3332 son la albañilería de adobe, tapial ${ }^{3}$, quincha ${ }^{4}$ y mampostería de piedra asentada en barro. Las expresiones arquitectónicas que quedan sujetas a ella son aquellas edificaciones construidas a base de tierra cruda, que hayan sido levantadas con anterioridad al 31 de julio de 1959, y cuyo propietario demuestre que esta construcción tiene, efectivamente, valor patrimonial. Este instrumento normativo está prestando un valioso aporte a los trabajos de protección y regularización del patrimonio construido en tierra cruda, protegido por la Ley 17.288 de Monumentos Nacionales, y se espera pueda servir en la prevención en intervenciones necesarias de acometer ante los ineludibles próximos desastres sísmicos.

Algunos de los alcances de esta norma: a) Establece con precisión los materiales, sistemas y elementos que abarca cada edificación, b) Instaura consideraciones en las intervenciones, es decir, criterios patrimoniales y estructurales, c) Establece una metodología para llevar a cabo el diagnóstico estructural de las construcciones, d) Establece los criterios para realizar el levantamiento de la estructura, e) Describe la metodología para realizar el análisis estructural y la determinación de esfuerzos frente a solicitaciones sísmicas mediante un método estático el cual define un coeficiente sísmico, considerando las condicionantes externas, f) Define pruebas de campo y valores básicos de comportamiento mecánico del material, y g) Caracteriza la intervención estructural de la construcción estableciendo bases de diseño y cálculo, reparación y refuerzo.

\footnotetext{
3 Técnica consistente en construir muros con tierra arcillosa, compactada a golpes mediante un "pisón", empleando un encofrado deslizante para contenerla.

${ }^{4}$ Entramado de caña o bambú recubierto con barro.
} 


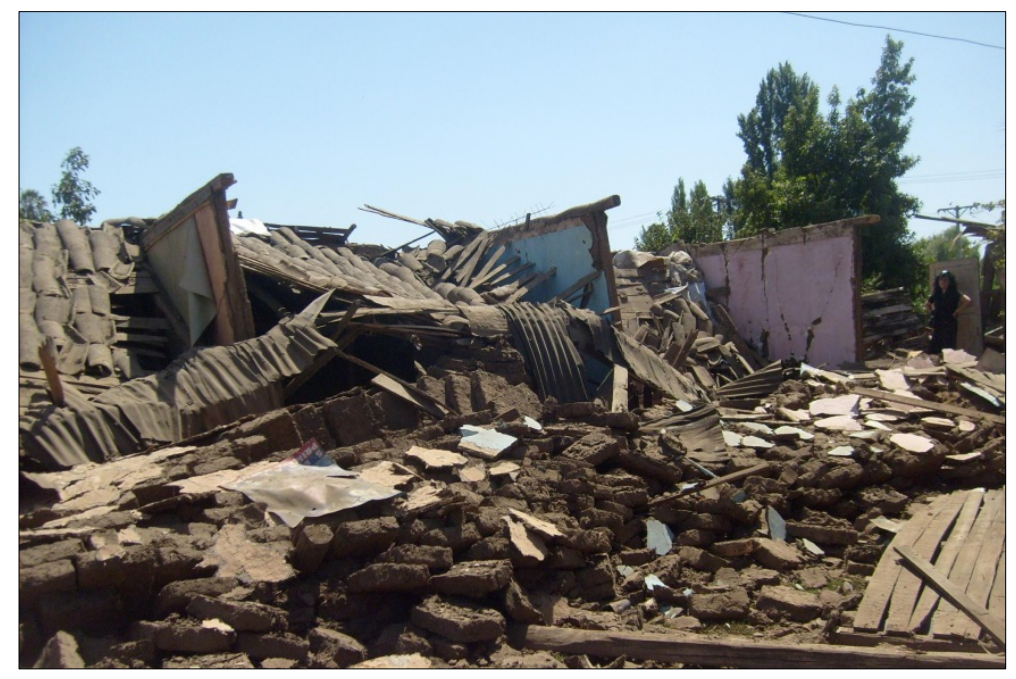

Fig. 7. Sismo de 2010 y la destrucción de la arquitectura construida en tierra cruda. La imagen corresponde a San Vicente de Tagua Tagua en la región del Libertador Bernardo O’higgins, al sur de la capital Santiago. Fuente: www.elmostrador.cl (consulta 06/03/2019).

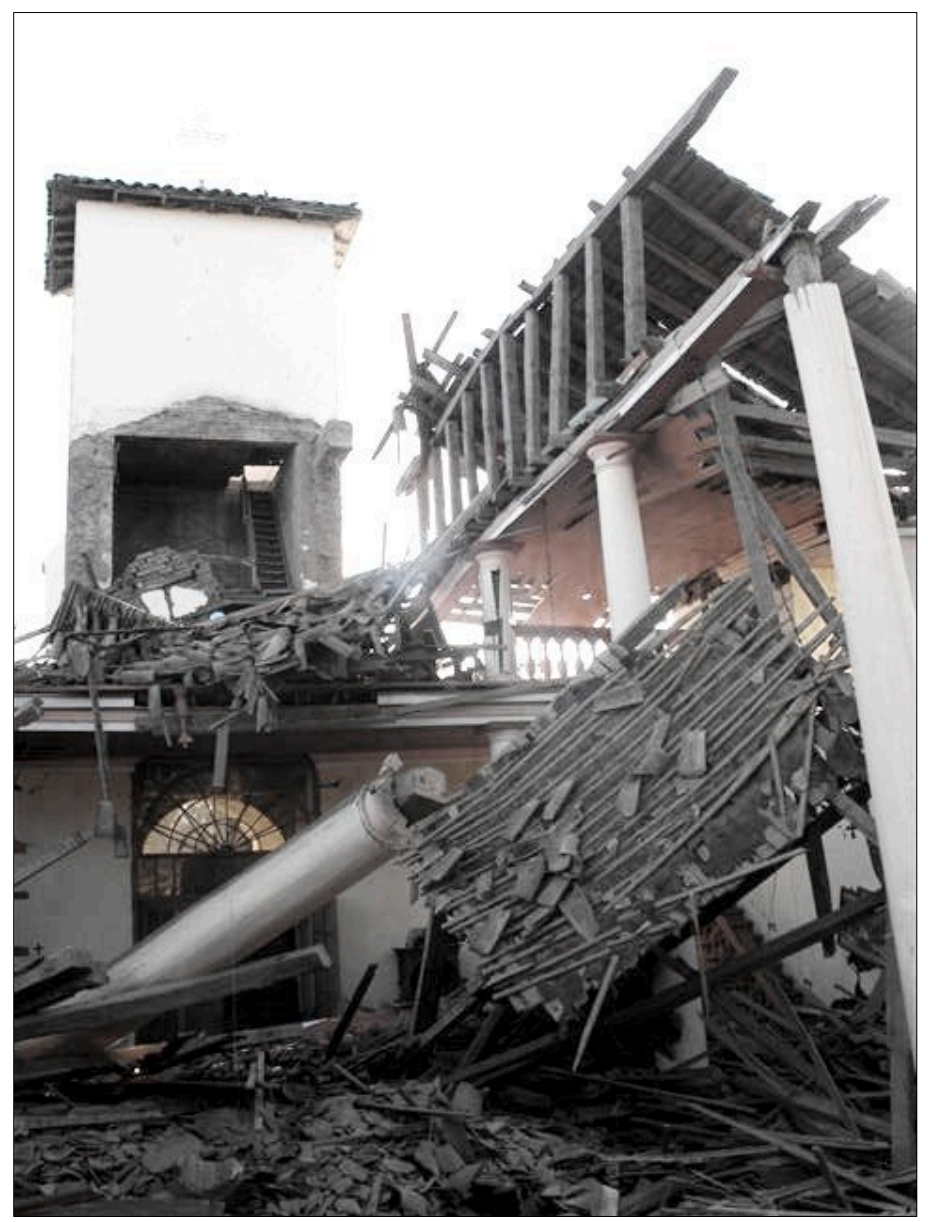

Figura 8. Iglesia San Antonio de Padua en la localidad de Chépica, región del Libertador Bernardo O’ higgins. Junto con la arquitectura habitacional, la religiosa experimentó daños de consideración. Fuente: www.plataformaarquitectura.cl, (consulta 06/03/2019)

\section{Conclusiones.}


Vista la realidad sísmica de Chile, puede decirse que desde el siglo XX y hasta lo que conocemos del XXI, se ha incrementado una buena respuesta ante las tareas de emergencia. Sin embargo, en lo que respecta al patrimonio cultural, y por extensión al arquitectónico y urbano, el Estado aún logra formular una política pública que se instale desde lo que significa la gestión de desastres sísmicos. La debilidad mayor se aprecia en el capítulo de la prevención y la planificación en el largo plazo. Lo común -que no lo ideal- es que se actúa reactivamente, cuando el fenómeno ya se produjo y, con ello, el desastre consiguiente.

Las secuelas del sismo-tsunami de 2010, si bien han permitido avances trascendentales como la oficialización de la Norma NCh 3332 de 2013, sobre construcciones a base de tierra cruda, en otros grandes temas del patrimonio arquitectónico, existen grandes tareas pendientes, sobre todo considerando la rica diversidad de expresiones de identidad existentes a lo largo del país.

Por ejemplo, no se conocen los procedimientos que permitan la reducción de su riesgo, a partir, por ejemplo, del control de los niveles de vulnerabilidad física y social presente en la arquitectura y los lugares de valor. Desde este enfoque, en Chile aún no se abordan integradamente los procesos de reconstrucción post sismo, que incorporen la variable del patrimonio, dependiendo de sus valores y atributos, respecto de sus aspectos físicos (forma, función y sistema constructivo), en conjunto con la revitalización de las comunidades.

En la Estrategia de Reducción de Riesgos, elaborada por la Oficina de las Naciones Unidas (2004), la gestión del riesgo ante desastres se define como "el manejo sistemático de decisiones administrativas, de organización, de capacidad técnica y de responsabilidades para la aplicación de políticas, estrategias y acciones para la reducción de riesgos ante desastres".

Los riesgos sísmicos que afectan al patrimonio nacional, debieran tener el mismo grado de importancia que los asociados a la urbanización, la presión inmobiliaria o turística. Los procesos de reconstrucción post desastres, considerando el patrimonio debieran concebirse "como un conjunto de procesos, priorizando el desarrollo sostenible y la activa participación de las comunidades afectadas" ICOMOS (2017).

Como señala ICOMOS (2017) la reconstrucción debiera observar tres fases: a) Identificación de los impactos recibidos por el patrimonio, en razón de sus valores y atributos materiales e inmateriales, b) Implementación de medidas de protección y estabilización de los atributos sobrevivientes, y c) Desarrollo de programas de recuperación y reconstrucción, destinados a transmitir a futuro los atributos de los bienes.

Debido a la naturaleza sísmica que enfrenta el patrimonio chileno, tanto Estado y la sociedad, debiéramos asegurarnos que los riesgos han sido correctamente identificados, que sus evaluaciones se actualizan periódicamente, considerando al mismo tiempo los aspectos materiales e inmateriales, y, que a partir de ello existan estrategias y planes de manejo, que consideren la mitigación de los riesgos que afecten los atributos del patrimonio.

Entre otros muchos factores, hay que poner el acento en los instrumentos legales, a fin de que toda nueva intervención en la edilicia -urbana o rural, nueva o antigua- lleve consigo, hasta donde sea posible y como condición sine quanon, el estudio antisísmico y las medidas preventivas asociadas. Se sabe que las construcciones antiguas no cumplen con los mínimos estándares de seguridad. También hay que actuar sobre la arquitectura contemporánea, para que, incluyendo el riesgo sísmico, desde su formulación y diseño, logre en el tiempo incrementar el acervo de identidades patrimoniales en la arquitectura chilena. 


\section{REFERENCIAS}

ARENAS, FEDERICO; HIDALGO, RODRIGO; LAGOS, MARCELO (2010). Los riesgos naturales en la planificación territorial. Centro de Políticas Públicas, Pontificia Universidad Católica de Chile, Instituto de Geografía, Año 5, N³9, octubre 2010.

CARDONA, OMAR DARÍO (2001) Estimación holística del riesgo sísmico utilizando sistemas dinámicos complejos. Tesis Doctoral. Universidad Politécnica de Cataluña.

CONSEJO INTERNACIONAL DE MONUMENTOS Y SITIOS. ICOMOS (2017). La recuperación y reconstrucción post trauma para propiedades culturales del Patrimonio Mundial. París, Consejo Internacional de Monumentos y Sitios (ICOMOS).

CONSEJO NACIONAL DE LA CULTURA Y LAS ARTES. CNCA (2005). Chile quiere más cultura. Definiciones de Política Cultural 2005-2010.Santiago de Chile.

CONSEJO NACIONAL DE LA CULTURA Y LAS ARTES. CNCA (2015). Política cultural 2011-2016. Primera edición. Santiago de Chile.

FERNÁNDEZ RICHARD, JOSÉ; HOLMES SALVO, FELIPE (2009). Derecho Urbanístico Chileno. Segunda edición actualizada. Santiago, Editorial Jurídica de Chile.

FERRADA, MARIO (2017). Eventos sísmicos, respuestas institucionales y producción de la arquitectura habitacional en Chile (1906-2010). Proyecto de investigación interno. Santiago, Facultad de Arquitectura y Urbanismo, Universidad de Chile.

GOBIERNO DE CHILE (2010). Plan de Reconstrucción. Terremoto y maremoto del 27 de febrero de 2010. Resumen Ejecutivo. 27 de agosto de 2010.

INSTITUTO DE LA CONSTRUCCIÓN DE CHILE (2013). Comisión de Construcción Patrimonial. Norma NCh 3332 Estructuras, Intervención de construcciones patrimoniales de tierra cruda y requisitos del proyecto estructural. 2013.

MINISTERIO DE VIVIENDA Y URBANISMO (2011). Plan de reconstrucción MINVU: Chile unido reconstruye mejor (cuarta edición). Santiago, Gobierno de Chile.

NACIONES UNIDAS, ONU (2009). UNISDR. Terminología sobre reducción de riesgos de desastres. Ginebra, Estrategia Internacional para la reducción de desastres de las Naciones Unidas (UNISDR).

NACIONES UNIDAS, ONU (2002). Message for the international day for disaster reduction "Disaster reduction for sustainable mountain development (October, $9 \quad$ 2002). Disponible en http://www.unisdr.org/2002/campaign/pa-camp02-sg-eng.htm (Consultado 02/03/2019).

OFICINA DE LAS NACIONES UNIDAS (2004). Estrategia Internacional para la Reducción de Desastres. Vivir con el Riesgo Informe mundial sobre iniciativas para la reducción de desastres. Ginebra, Suiza, Naciones Unidas.

VARGAS, JORGE ENRIQUE (2002). Políticas Públicas para la reducción de la vulnerabilidad frente a los desastres naturales y socio- naturales. CEPAL, División de Medio Ambiente y Asentamientos Humanos. Serie Medio Ambiente y Desarrollo, Santiago de Chile, 2002. 